\title{
New approach to interpret Short-offset transient electromagnetic (SOTEM) data
}

\author{
Muhammad Younis Khan ${ }^{1,2}$, Guoqiang Xue ${ }^{1}$, Weiying Chen ${ }^{1}$, Huasen Zhong ${ }^{1}$ \\ Key Laboratory of Mineral Resources \\ Institute of Geology and Geophysics,CAS \\ Beijing, China \\ National Centre of Excellence in Geology \\ University of Peshawar \\ Khyber Pakhtunkhwa, Pakista
}

\begin{abstract}
Commonly, TEM data is plotted as apparent resistivity versus time. Later on, with improvement in computer technology, inversion programs have got attention of EM geophysical community. Recently, inversion routines are applied to 1-D problems. In this paper we propose a new approach to interpret SOTEM data by carrying out analysis in time, frequency and pseudo-seismic domain. At first, we processed synthetic data for $H \& K$ type models using 1-D Occam inversion. Then we transformed time-domain data into quasi-MT domain and conducted 1-D Bostick's algorithm. Finally, EM diffusion field is transformed into wave field reflecting seismic-like response with some reflection and refraction phenomena. Our proposed approach is validated by a case study of a coal field located in Henan province, China.
\end{abstract}

Keywords-interpretation; SOTEM; transient electromagnetic method

\section{I.INTRODUCTION}

The short-offset transient electromagnetic method (SOTEM) is a type of transient electromagnetic sounding method where the offset between transmitter and receiver is approximately set equal to or greater than the target depth. However, to cover a large area, particularly, the offset of SOTEM measurements conducted in rough and complex mountainous regions may be extended to two times that of the sounding depth. Like other TEM methods, the SOTEM has been recognized as promising TEM version applied in in a variety of hydrogeological investigation, mineral and hydrocarbon exploration ${ }^{[1,3,9,14]}$.

The final task of EM response in time/frequency domain is to study electrical structure by transformation of voltage into apparent resistivity which is most common variable in spite of difference in theoretical concepts of both EM methods. However using Fourier technique we can switch the data from TEM to FEM. By comparing resistivity curves of central loop TEM and MT, XUE et al. 2006 proposed a time-frequency (T-F) relationship in range of $\mathrm{f}=250 / \mathrm{t} \sim \mathrm{f}=$ $119.2 / \mathrm{t}(\mathrm{ms})$ and an optimal relation of $\mathrm{f}=210 / \mathrm{t}$ which allow us to apply quais-MT inversion method to transformed SOTEM results. In last, we transformed SOTEM data into pseudo-seismic.

\section{II.APPARENT RESISTIVITY}

Bisection approach based on simple computational routine to solve nonlinear equations has been extensively applied $^{[6]}$ where EM field response must need to be monotonic with resistivity. Therefore, measured voltage is transformed to magnetic field using (1).

$$
H_{z}(t)=\frac{1}{S \mu_{0}} \int_{t}^{b} V_{z}(t) d t+H_{z}(b)=\frac{1}{S \mu_{0}} \int_{t}^{b} V_{z}(t) d t
$$

Where, $S$ is the effective areas of induced coil, $\mu_{0}$ is the permeability of the air, $H_{z}(b)$ is the magnetic field of the last time channel.

To fit the observed data, different resistivity values are substituted in (2).

$$
H_{z}(t)=\frac{I d l}{4 \pi r^{2}} \sin \varphi\left[\frac{3}{\sqrt{\pi}} \frac{e^{-u^{2}}}{u}+\left(1-\frac{3}{u^{2}}\right) \Phi(u)\right]
$$

Where, $I$ is the transmitting current, $d l$ is the length of dipole source, $r=\sqrt{x^{2}+y^{2}}$ is the offset, $\varphi$ is the angle between receivers and transmitter, $u=\frac{2 \pi r}{\tau}$, $\tau=2 \pi \sqrt{2 \rho t / \mu_{0}}=\sqrt{2 \pi \rho t \times 10^{7}} \quad, \quad \phi(u)=\sqrt{\frac{2}{\pi}} \int_{0}^{u(t)} e^{-t^{2} / 2} \mathrm{~d} t \quad$ is the probability function.

CAGNIARD (1953) gave a (3) used for estimation of apparent resistivity in MT method.

$$
\rho^{M T}=\frac{1}{5 f}\left|\frac{E_{x}}{H_{y}}\right|^{2}
$$

Where, $f$ is frequency, $E_{x}$ is the horizontal electric field parallel to source, and $H_{y}$ is the horizontal magnetic field perpendicular to source. 


\section{III.OCCAM INVERSION, T-F TRANSFORMATION \& QUASI- MT INVERSION OF SOTEM DATA}

Occam's 1D inversion routine ${ }^{[2]}$ were applied to SOTEM data (Fig.1). Different T-F transformation schemes have been introduced by ${ }^{[4,5,10]}$ analyzed the similarity of apparent resistivity curves in two domains at optimal level. So firstly, we converted STEM data using the relation $f=133.4 / t$. Then, we tested simplest Bostick inversion technique ${ }^{[7]}$ to get results in frequency domain (Fig.2).

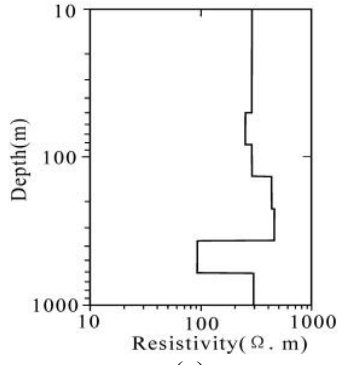

(a)

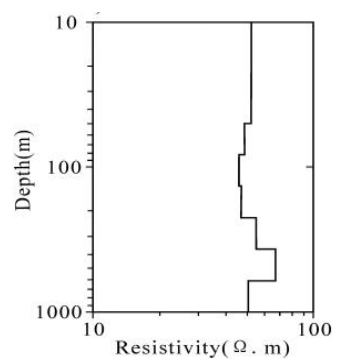

(b)

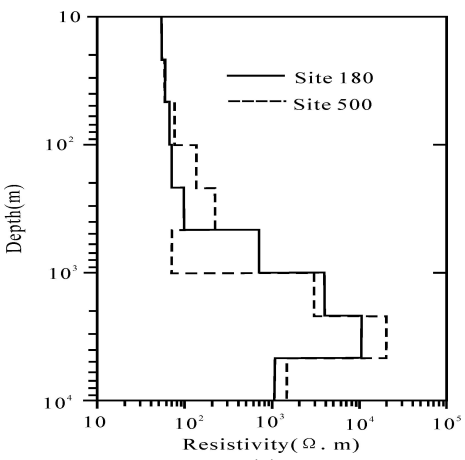

(c)

Fig.1. 1-D Occam inversion results (a) H- type (b) K- type (c) real data

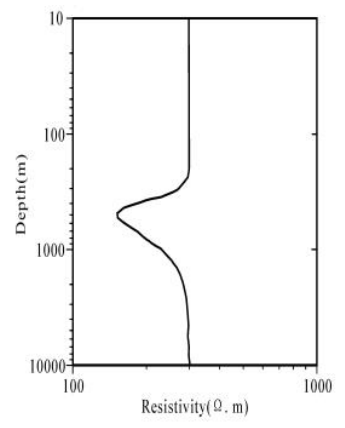

(a)

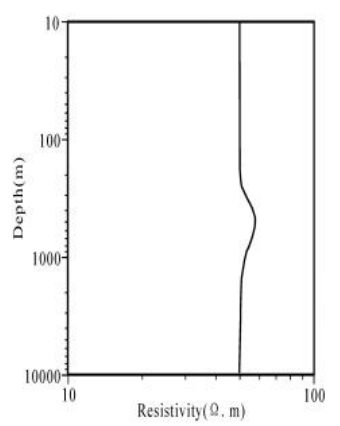

(b)

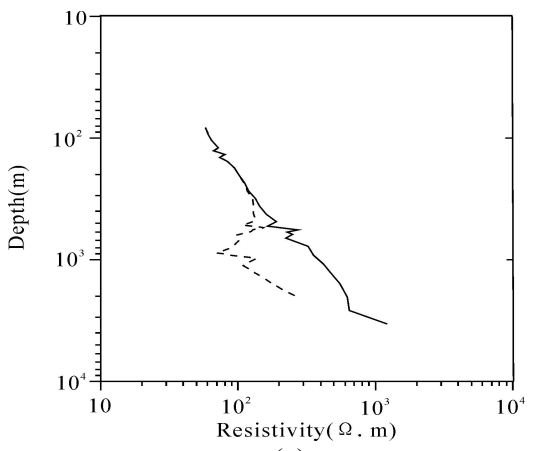

(c)

Fig.2. 1D Bostick inversion results of two-layer models H-type (a) , Ktype (b) and (c) real data

\section{IV.PSEUDO-SEISMIC EQUIVALENT OF SOTEM DATA}

SOTEM data is converted to seismic-like (pseudoseismic) time section (Fig.3), using special (4) ${ }^{[8]}$.

$$
H_{m}\left(t_{i}\right)=\sum_{j=1}^{n} u\left(\tau_{j}\right) a\left(t_{i}, \tau_{j}\right) h_{j}
$$

Where, $a\left(t_{i}, \tau_{j}\right)=\frac{1}{2 \sqrt{\pi t_{i}^{3}}} \tau_{j} e^{\frac{\tau_{j}^{2}}{4 t_{i}}}, h_{j}$ is the integral coefficient and $H_{m}(t), U(\tau)$ is diffusion field and the pseudowave field, respectively.

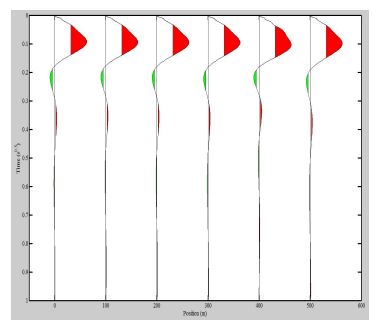

(a)

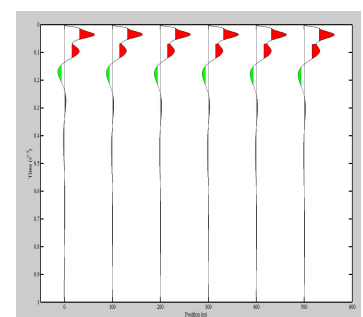

(b)

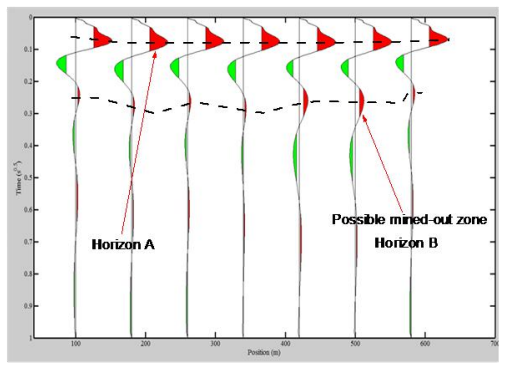

(c)

Fig.3. Pseudo-seismic results of two-layer models H-type (a) , K-type (b) and (c) real data 
Fig.1 \& Fig.2 showed that like synthetic data results, conductive anomaly was resolved clearly in case of both time and frequency domain inversion schemes around $1000 \mathrm{~m}$. However, quasi-MT results were not very appreciable in retrieving the anomalous zone (excavated water filled zones) underlain and overlain by sandstone beds in the survey area. Pseudo-seismic section clearly indicated the target zone which can also be correlated to 1-D inversion results within same depth interval ranging from $450-1000 \mathrm{~m}$.

\section{V.CONCLUSIONS}

First, synthetic and real data were analyzed in timedomain by popular Occam inversion in time-domain which indicated a low conductivity anomaly within 450-100m, which is then verified by subsequent quasi MT inversion, pseudo-seismic and drilling results.

Interpretation of SOTEM field data in different domains provides an alternative way to explore the TEM soundings further in order to improve our understanding by introducing new interpretation methodology.

\section{REFERENCES}

[1] CHEN, W. Y., G. Q. XUE, K. M. YOUNIS 2015 Application of Short-Offset TEM (SOTEM) Technique in Mapping Water-Enriched Zones of Coal Stratum, an Example from East China: Pure and Applied Geophysics, 172 (6): 1643-1651.

[2] CAGNIARD, L1953 Basic theory of the magnetotelluric method of geophysical prospecting: Geophysics, 18, 605-635.

[3] LI, H., G. Q. XUE, N. N. ZHOU, AND W. Y. CHEN 2015 Appraisal of an array TEM method in detecting a mine-out area beneath a conductive layer: Pure and Applied Geophysics, 172(10): 2917-2929.
[4] Meju, M. A.. 1995. Simple effective resistivity-depth transformations for infield or real-time data processing: Computers \& geosciences, 21(8): 985-992.Meju, M. A..1996. Joint inversion of TEM and distorted MT soundings: some effective practical considerations. Geophysics, 61(1): 56-65.

[5] Meju, M. A.. 1998. A simple method of transient electromagnetic data analysis. Geophysics, 63(2): 1-6.

[6] PRESS, W. H., S. A. TEUKOLSKY, W. T. VELlERLING., B. P. FLANNERY. 1992 Numerical recipes in Fortran: Cambridge Press

[7] WEIDELT, P. 1972 The inverse problem of geomagnetic induction: Zeit. fu ${ }^{\circ}$ r Geophys., 38, $257-298$.

[8] XUE G Q, YAN Y J AND LI X 2007 Pseudo-seismic wavelet transformation of transient electromagnetic response in engineering geology exploration Geophys. Res. Lett. 34 L16405.

[9] Xue, G. Q., L. J. Gelius, P. A. Sakyi, et al., 2014, Discovery of a hidden BIF deposit in Anhui province, China by integrated geological and geophysical investigations: Ore Geology Reviews, 63, 470-477

[10] Xue, G. Q., Li, X., Guo, W. B., et al., 2006, Equivalent transformation from TEM field sounding data to plane-wave electromagnetic sounding data, Chinese journal of Geophysics (in Chinese), 49, 1539-1545.

[11] XUE G Q, yan y j., li x 2011 Control of the waveform dispersion effect and applications in a TEM imaging technique for identifying underground objects J. Geophys. Eng. 8195 - 201.

[12] ZHDANOV, M. S 2010 Electromagnetic geophysics: Notes from the past and the road ahead Geophysics (75): A49-75A66 .

[13] ZHOU, N. N., G. Q. XUE, W. Y. CHEN., J. Cheng 2015 Largedepth hydrogeological detection in the North China-type coalfield through short-offset grounded-wire TEM: Environmental Earth Sciences, 74, 2393-2404.

[14] ZIOLKOWSKI, A., B. A. HOBBS., D. WRIGHT 2007 Multitransient electromagnetic demonstration survey in France: Geophysics, 72(4): F197-F209. 